\title{
Indução do parto em gestantes no pós-datismo no estado do Piauí
}

\author{
Induction of childbirth in postpartum pregnant women in the state of Piauí \\ Inducción del parto en mujeres embarazadas posparto en el estado de Piauí
}

Francisca Jéssica de Sousa Oliveira ${ }^{1 *}$, Daylana Braga Soares ${ }^{1}$, Priscila Silva Gaspar ${ }^{1}$, Ana Márcia Morais do Nascimento Almeida ${ }^{1}$, Karla Joelma Bezerra Cunha².

\section{RESUMO}

Objetivo: Analisar as induções ao trabalho de parto no pós-datismo; estudo ocorrido na sala de parto e alas de internação de uma maternidade de referência no atendimento de urgência/emergência em capital no estado do Piauí. Métodos: Pesquisa descritiva exploratória, quantitativa. Coleta dos dados através de formulário e utilizou-se o software Stata®, v.12 (Statacorp, College Station, Texas, USA) para a organização e análise dos dados utilizando a associação entre as variáveis pelo teste Qui-quadrado de Pearson $\left(\mathrm{x}^{2}\right)$ ou teste exato de Fisher quando apropriado. Resultados: Foram obtidos 56 casos concluindo-se o perfil das mulheres sendo solteiras, de baixa escolaridade e renda, de ocupação doméstica. No que se refere à paridade das pacientes, o estudo revelou que a maioria delas eram primigestas, nulíparas e realizaram parto cesariano, com associação significativa entre as variáveis em relação ao tipo de parto. Evidenciaram o parto Cesário nas induções com misoprostol devido à frequência de falha de indução, podendo considerar que as variáveis posição do colo posterior e primiparidade foram estatisticamente significantes. Conclusão: Foi observada a necessidade da existência de um protocolo a ser seguido por toda a equipe envolvida no processo de indução, pois o resultado mostrou que a maioria delas apresentaram falhas, fato que pode estar relacionado ao desfavorecimento do colo na posição posterior.

Palavras-Chave: Trabalho de parto induzido, Misoprostol, Ocitocina.

\begin{abstract}
Objective: To analyze induction of labor in post-datism; this study was carried out in the delivery room and hospitalization wards of a reference maternity ward in the state of Piauí. Methods: Exploratory, quantitative descriptive research. Data were collected using the form and Stata® software, v.12 (Statacorp, College Station, Texas, USA) was used to organize and analyze the data using the association between variables by Pearson's chi-square test $\left(x^{2}\right)$ or Fisher's exact test when appropriate. Results: We obtained 56 cases, concluding the profile of women being single, with low education and income, of domestic occupation. Regarding the parity of the patients, the study revealed that most of them were primiparous, nulliparous and underwent cesarean delivery, with a significant association between the variables regarding the type of delivery. They showed cesarean delivery in induction with misoprostol due to the frequency of induction failure and may consider that the variables posterior neck position and primiparity were statistically significant. Conclusion: It was observed the need for a protocol to be followed by all the team involved in the induction process, because the result showed that most of them had flaws, a fact that may be related to the disadvantage of the neck in the posterior position.
\end{abstract}

Key words: Induced labor, Misoprostol, Oxytocin.

\footnotetext{
${ }^{1}$ Centro Universitário Santo Agostinho (UNIFSA), Teresina-PI. *E-mail: fjessica_pi100@hotmail.com

${ }^{2}$ Universidade do Vale do Paraíba/Instituto de Pesquisa e Desenvolvimento, São José dos Campos - São Paulo.
} 


\section{RESUMEN}

Objetivo: Analizar la inducción del parto en el postdatismo; este estudio se realizó en la sala de partos y salas de hospitalización de una sala de maternidad de referencia en el estado de Piauí. Métodos: Investigación exploratoria, cuantitativa descriptiva. Los datos se recopilaron utilizando el formulario y el software Stata $\AA_{\text {, }}$ v.12 (Statacorp, College Station, Texas, EE. UU.) Se utilizó para organizar y analizar los datos utilizando la asociación entre variables mediante la prueba de chi-cuadrado de Pearson $\left(x^{2}\right)$ o la prueba exacta de Fisher cuando corresponda. Resultados: Obtuvimos 56 casos, concluyendo el perfil de mujeres solteras, con baja educación e ingresos, de ocupación doméstica. Con respecto a la paridad de los pacientes, el estudio reveló que la mayoría de ellos eran primíparos, nulíparos y se sometieron a cesárea, con una asociación significativa entre las variables con respecto al tipo de parto. Mostraron cesárea en la inducción con misoprostol debido a la frecuencia de falla de la inducción, y pueden considerar que las variables posición posterior del cuello y primiparidad fueron estadísticamente significativas. Conclusión: Se observó la necesidad de que un protocolo sea seguido por todo el equipo involucrado en el proceso de inducción, porque el resultado mostró que la mayoría de ellos tenían fallas, un hecho que puede estar relacionado con la desventaja del cuello en la posición posterior.

Palabras clave: Trabajo inducido, Misoprostol, Oxitocina.

\section{INTRODUÇÃO}

A gestação é um fenômeno fisiológico que, em sua maior parte, evolui sem intercorrências. Segundo observações clínicas e as estatísticas, a maioria das gestações iniciam, evoluem e terminam sem complicações, denominadas como gestações de risco habitual; outras, já se iniciam com problemas ou estes surgem durante o seu percurso e apresentam desfechos possivelmente desfavoráveis, sejam eles para o feto ou para a mãe, classificando-se como gestação de alto risco (FREITAS F, et al., 2011).

Menos de 10\% das gestações encerram na data provável do parto (DPP), apesar de a maioria dos nascimentos ocorrer em torno do prazo de 7 dias da DPP. Nos Estado s Unidos, cerca de $13 \%$ dos nascimentos acontecem antes das 37 semanas de gestação, e cerca de 5 a $7 \%$ aguardam o parto até depois de 42 semanas de gestações, ou seja, 14 dias após a DPP, conhecida como pós-termo. Essas taxas são mais baixas para as gestações adequadamente datadas (CHESTNUT D, et al., 2016).

Segundo Chaves Netto H e Sá RAM (2015) são facilmente reconhecíveis as manifestações adaptativas da mulher durante a gestação, sendo elas de grande importância para o diagnóstico e avaliação da mesma. O diagnóstico clínico de gravidez é feito com base em sinais e sintomas referidos durante a anamnese e exame físico, sendo agrupada a todas as informações coletadas a análise dos sintomas de presunção e sinais de probabilidade e certeza de gravidez, que se atesta pelo resultado $\mathrm{d}$ os testes laboratoriais.

As alterações fisiológicas observadas na gestação são decorrentes, principalmente, de fatore s hormonais e mecânicos e os ajustes verificados no organismo da mulher deve $\mathrm{m}$ ser considerados normais durante 0 estado gravídico, embora determine, por vez es, pequenos sintomas que afetam a saúde da paciente (MONTENEGRO CAB e REZENDE FILHO J, 2015).

Segundo Carvalho SA (2013), o trabalho de parto é o processo fisiológico em que o útero, por meio de contrações naturais e involuntárias, expulsa o feto, a placenta e as membranas para o seu exterior através do canal vaginal em mulheres com idade gestacional igual ou superior a 20 semanas. Em relação à duração, pode ser pré-termo, se iniciado de 20 a 36 semanas e 6 dias de gestação, a termo, se a gestação é de 37 a 41 semanas e 6 dias, e pós-termo, nas gestantes com idade gestacional superior a 42 semanas.

O período gestacional classifica-se, conforme sua duração, em prematuro, todo concepto nascido entre 20 e 37 semanas; gravidez normal, que deva terminar entre 38 e 42 semanas; pós-datismo, quando a gestação transcende 40 semanas; prolongada, que excede 42 semanas, sem comprometimento do bem-estar fetal; pós-maturidade, quando ultrapassa o período estimado cursando com sofrimento fetal e insuficiência 
placentária. Entretanto, vários são os termos utilizados para a gestação prolongada, entre eles pós-termo, gestação tardia, gravidez serotínea e pós-data. O conceito é variável, pois a literatura é duvidosa quando se considera pelo tempo de duração da gestação (CHAVES NETTO H e SÁ RAM, 2015).

De acordo com Freitas F, et al. (2011), o manejo da gestação pós-termo é ainda um problema na prática obstétrica. A incidência da gestação pós-termo vai de 4 a $14 \%$ das gestações e varia de acordo com a definição utilizada e com o percentual de pacientes com erro de avaliação da idade gestacional. Esse erro de estimativa é menor se a idade gestacional é calculada por ecografia realizada no primeiro trimestre, com comparação a ecografia realizada no segundo trimestre. $O$ ultrassom realizado precocemente proporciona uma estimativa mais correta que a idade gestacional, reduzindo a taxa de falso pós-datismo que reduz intervenções desnecessárias.

A gravidez pós-termo verdadeira ainda tem causa desconhecida. Entretanto, está claro que o momento do trabalho de parto é determinado por interações completas entre o feto, a placenta e as membranas, o miométrio uterino e a cérvice. Desta forma, quando não ocorrem estas interações, são necessárias intervenções, sendo uma a indução ao trabalho de parto (LOWDERMILK DL, et al., 2012).

Conforme Ricci SS (2015), de forma ideal, todas as gestações vão até o termo, iniciando o trabalho de parto espontaneamente. No entanto, muitas gestantes precisam de ajuda para iniciar ou sustentar este processo; a indução do trabalho de parto envolve estimular as contrações uterinas por meios clínicos ou cirúrgicos antes do início espontâneo do mesmo, sendo este um evento não isolado por trazer muitas outras intervenções que podem ou não produzir um desfecho favorável.

Desde 1991, a indução do trabalho de parto é um dos procedimentos obstétricos mais comuns; mais do que duplicou (CHENG YW, et al., 2012). Indução do parto é a iniciação química ou mecânica de contrações uterinas antes de seu início espontâneo, objetivando realizar o parto, sendo este induzido seletivamente ou por razões indicadas, o método indicado, se o desenvolver da gestação apresentar risco para a mãe e/ou o feto, e se não existirem contraindicações à ruptura artificial das membranas (amniotomia) ou aumento das contrações uterinas com ocitocina, assim, antes da iniciativa do procedimento, deve ser verificada a idade gestacional e avaliar riscos potenciais ao binômio (LOWDERMILK DL, et al., 2012).

O objetivo da pesquisa ao analisar as induções é que essa pesquisa possa contribuir com informações sobre o processo de indução em gestantes com pós-datismo, e que possa nortear novas pesquisas servindo de reflexão aos profissionais sobre uma prática mais eficaz baseada em evidências científicas.

\section{MÉTODOS}

O estudo ocorreu na sala de parto e alas de internação em uma maternidade de referência voltada para atendimento de urgência/ emergência obstétrica e gestações de alto risco referência do estado do Piauí, localizada na zona sul da capital.

A amostra foi composta por 56 mulheres internadas no centro obstétrico com diagnóstico de trabalho de parto tendo como critérios de inclusão mulheres a partir de 40 semanas de gestação, aquelas com indicação para o processo de indução ao trabalho de parto, internadas no período proposto da pesquisa, que concordaram e assinaram o Termo de compromisso livremente esclarecido (TCLE), mediante esclarecimento sobre os objetivos do estudo; as mesmas foram abordadas em momento conveniente para a parturiente e seu acompanhante.

Foram excluídas aquelas que não concordaram em participar do mesmo e/ou não assinaram o TCLE, e as que possuíam transtornos mentais, apresentação fetal pélvica e as que foram internadas fora do período proposto da pesquisa. Para realizar a coleta dos dados, foi utilizado um formulário semiestruturado, com perguntas abertas e fechadas, sendo utilizado para contribuir e completar as informações, os prontuários e cartão da gestante, respeitando os objetivos da pesquisa. A coleta de dados procedeu-se nos meses de setembro e outubro de 2017. Contempladas as variáveis sociodemográficas como: faixa etária, cor da pele, estado civil, nível de escolaridade, procedência, ocupação, renda, antecedentes obstétricos como: paridade, duração da gestação, idade da menarca, consistência e posição do colo uterino. 
Utilizou-se o software Stata®, v.12 (Statacorp, College Station, Texas, USA) para a organização e análise dos dados. As variáveis foram apresentadas por meio de estatística descritiva: número e proporções. Testouse a associação entre as variáveis pelo teste Qui-quadrado de Pearson $\left(x^{2}\right)$ ou teste exato de Fisher quando apropriado. Foram aceitos como estatisticamente significativos os testes com valor de $p<0,05$. A pesquisa foi submetida para a apreciação pela Comissão de Ética; submetido a Plataforma Brasil e enviado ao comitê de ética do centro de ensino responsável para apreciação, tendo o número do parecer: 2.225.766. Comitê de Ética em Pesquisa (CAAE): 72313717. 1. 0000. 5602.

\section{RESULTADOS E DISCUSSÃO}

Estudar as induções ao trabalho de parto em gestações que ultrapassam 40 semanas instiga ao desejo de entender por que essas pacientes não evoluem para trabalho de parto espontâneo e o que pode ser feito para que elas iniciem esse trabalho de parto de forma mais fisiológica, e como esse processo acontece. Acredita-se que a análise das induções pode fornecer informações relevantes na busca da diminuição dos números de cesáreas eletivas.

Foram obtidos 56 casos que atendiam aos critérios do estudo. Os dados foram organizados em tabelas e caracterizados de acordo com as variáveis: aspectos sociodemográficos e econômicos, antecedentes ginecoobstétricos, tipo de indução, fármacos utilizados, assim como a via de administração, dose, intervalo entre as doses, o tempo de indução, desfecho final e a justificativa do desfecho. A seguir temos a Tabela 1 com a descrição das características sociodemográficas e econômicas por tipo de parto.

Tabela 1 - Descrição das características sociodemográficas e econômicas por tipo de parto. Teresina-PI, $2017(\mathrm{~N}=56)$.

\begin{tabular}{|c|c|c|c|c|c|}
\hline Variáveis & \multicolumn{2}{|c|}{ Parto Cesário } & \multicolumn{2}{|c|}{ Parto Normal } & Valor $p$ \\
\hline \multicolumn{6}{|l|}{ Idade (anos) } \\
\hline$\leq 19$ & 13 & 23,3 & 4 & 7,1 & \multirow{3}{*}{$0,386^{*}$} \\
\hline 20 a 29 & 15 & 26,8 & 12 & 21,4 & \\
\hline$\geq 30$ & 8 & 14,3 & 4 & 7,1 & \\
\hline \multicolumn{6}{|l|}{ Cor da pele } \\
\hline Branca & 4 & 7,1 & 4 & 7,1 & \multirow{3}{*}{$0,669^{*}$} \\
\hline Negra & 9 & 16 & 5 & 9,0 & \\
\hline Parda & 23 & 41,2 & 11 & 19,6 & \\
\hline \multicolumn{6}{|l|}{ Estado civil } \\
\hline Solteiro & 26 & 46,4 & 8 & 14,3 & \multirow{4}{*}{$0,016^{*}$} \\
\hline Casado & 2 & 3,5 & 5 & 9,0 & \\
\hline Mora com companheiro & 8 & 14,3 & 5 & 9,0 & \\
\hline Termo de união estável & - & - & 2 & 3,5 & \\
\hline \multicolumn{6}{|l|}{ Escolaridade } \\
\hline Analfabeto & 1 & 1,8 & - & - & \multirow{4}{*}{$0,054^{*}$} \\
\hline Ens. Fundamental & 30 & 53,6 & 12 & 21,4 & \\
\hline Ens. Médio & 5 & 9,0 & 6 & 10,7 & \\
\hline Ens. Superior & - & - & 2 & 3,5 & \\
\hline \multicolumn{6}{|l|}{ Procedência } \\
\hline Teresina (urbana) & 20 & 35,8 & 11 & 19,6 & \multirow{3}{*}{$0,781^{*}$} \\
\hline Urbana & 13 & 23,3 & 6 & 10,7 & \\
\hline Rural & 3 & 5,3 & 3 & 5,3 & \\
\hline \multicolumn{6}{|l|}{ Ocupação } \\
\hline Salariada & 2 & 3,5 & 2 & 3,5 & \multirow{3}{*}{$0,887^{\star}$} \\
\hline Ocupaçao domestica & 28 & 50 & 15 & 26,8 & \\
\hline Estudante & 6 & 10,7 & 3 & 5,3 & \\
\hline \multicolumn{6}{|l|}{ Renda (salário mínimo) } \\
\hline Até 1 & 33 & 58,9 & 16 & 28.5 & \\
\hline 1 a 3 & 3 & 5,3 & 4 & 7,1 & $0,234^{*}$ \\
\hline
\end{tabular}

Legenda: *Teste exato de Fisher. Fonte: Oliveira FJS, et al., 2017 
O estudo evidenciou que $26,8 \%$ das pacientes foram submetidas ao processo de indução e estavam na faixa etária de 20 a 29 anos; 41,2\% eram pardas e evoluíram para parto cesariano. Em relação à escolaridade, observou-se que as pacientes que realizaram parto cesariano tinham como escolaridade o nível fundamental, com $53,6 \%$.

Essas variáveis apesentam significância em relação ao tipo de parto. Referindo-se ao estado civil, a amostra mostrou associação estatisticamente significativa em relação ao tipo de parto, em que prevaleceu o parto cesariano em mulheres solteiras, com $46,4 \%$.

Mulheres com ocupação "domésticas" foram mais frequentes na amostra, com 50\%; a renda familiar foi de até um salário mínimo, encontrada em $58,9 \%$ das participantes; estas evoluíram para parto Cesário, apesar de não mostrar correlação significativa entre as variáveis. Das gestações intituladas com pós-datismo que foram submetidas à indução do parto, 56 casos se adequaram aos critérios da pesquisa.

A faixa etária prevalente das gestantes foi de 20 a 29 anos, sendo que estas mulheres em idade adulta jovem evoluíram para parto cesário, resultado que contradiz com o estudo de Knupp VMA, Melo ECP e Oliveira RB (2008), o qual afirmam que quanto maior a idade da mãe, maior a taxa de parto cesário.

A etnia prevalente neste estudo foi a raça parda, que, de acordo com os dados do IBGE (2010), é predominante no estado do Piauí-PI, representando $64 \%$ da população geral; estes dados justificam a prevalência dessa etnia na pesquisa, com $63,9 \%$ dos casos de cesária, apesar de não mostrar correlação significativa com o tipo de parto. Um estudo transversal abordando comparativamente 163 primíparas de uma maternidade pública e 89 de uma maternidade privada, com médias de idade de 21 anos com gestação pósdatista realizado no ano de 2007 por Mandarino NR et al. (2009) em São Luiz-MA, relacionaram aspectos que evidenciaram a cor parda, com taxas elevadas de mulheres atendidas na rede pública, com $57 \%$.

Em relação ao estado civil das mulheres submetidas ao processo de indução, prevaleceram as solteiras. Queiroz MV et al. (2005) evidenciaram, em seu estudo, que as mulheres não brancas e solteiras tinham maior probabilidade de terem partos normais, enquanto mulheres mais velhas e casadas tinham a probabilidade de terem partos cesários, se contrapondo aos resultados desse estudo, o que já era esperado, por fazer parte de realidades temporais diferentes.

Tratando-se dos tempos atuais, Moreira BR, et al. (2016) publicaram um estudo observacional transversal comparativo entre o número de partos vaginais e cesarianas de 82 mulheres atendidas na Fundação Vespasianense de Saúde de agosto de 2012 a outubro de 2013, onde obteve como resultado a maior porcentagem de partos cesários em mulheres solteiras, enquanto que as casadas tiveram parto normal. De acordo com Queiroz MV, et al. (2005) o tipo de parto mediante estado civil pode ser influenciado por fatores psicológicos e preocupações com o futuro.

De acordo com a característica econômica, a renda das mulheres era de até um salário mínimo. Girotto LC, et al. (2013) chamam a atenção para as desigualdades sociais, pois as condições de vida às quais as gestantes estão submetidas exercem uma grande influência nas condições da gestação. Baixa escolaridade, pouca profissionalização, assim como abandono do parceiro ou da família, são fatores que interferem em todo o processo da gestação.

A pesquisa mostrou que $30,3 \%$ das mulheres que tiveram menarca aos treze anos evoluíram para parto cesariano. No que se refere à paridade das pacientes, o estudo revelou que $57,1 \%$ delas eram primigestas, nulíparas e realizaram parto cesariano, com associação significativa entre as variáveis. No que se refere à gestação atual, $57 \%$ das gestações apresentaram duração entre 40 a 42 semanas, com colo uterino de consistência mole em $32.1 \%$ dos casos, com posição posterior em $41.2 \%$, e a posição do colo mostrou associação estatisticamente significativa com o tipo de parto.

A descrição das variáveis dos antecedentes ginecológicos e obstétricos e os da gestação atual como: idade da menarca, número de gestações, aborto, duração da gestação atual, consistências do colo uterino e sua posição, são variáveis importantes na tentativa de compreender o processo fisiológico da indução e seus desfechos. Estas variáveis foram verificadas neste estudo, conforme os dados que seguem (Tabela 2). 
Tabela 2 - Descrição dos antecedentes gineco-obstétricos e dados da gestação atual por tipo de parto. Teresina-PI, 2017 ( $\mathrm{N}=56)$.

\begin{tabular}{|c|c|c|c|c|c|}
\hline Variáveis & $\begin{array}{l}\text { Parto Cesário } \\
\text { № }\end{array}$ & $\%$ & $\begin{array}{l}\text { Parto Normal } \\
\text { № }\end{array}$ & $\%$ & Valor $p$ \\
\hline \multicolumn{6}{|l|}{ Idade da menarca (anos) } \\
\hline 11 & 4 & 7,1 & 3 & 5,3 & \multirow{5}{*}{$0,474^{*}$} \\
\hline 12 & 10 & 17,8 & 8 & 14,4 & \\
\hline 13 & 17 & 30,3 & 6 & 10,7 & \\
\hline 14 & 5 & 9,0 & 2 & 3,5 & \\
\hline 15 & - & - & 1 & 1,8 & \\
\hline \multicolumn{6}{|l|}{ № de gestações } \\
\hline 1 & 30 & 53,6 & 9 & 16 & \multirow{3}{*}{$0,012^{*}$} \\
\hline 2 a 3 & 4 & 7,1 & 7 & 12,5 & \\
\hline 4 ou mais & 2 & 3,5 & 4 & 7,1 & \\
\hline 0 & 32 & 57,1 & 9 & 16 & \multirow{3}{*}{$0,001^{*}$} \\
\hline 1 & 3 & 5,3 & 4 & 7,1 & \\
\hline$\geq 2$ & 1 & 1,8 & 7 & 12,5 & \\
\hline \multicolumn{6}{|l|}{ № de aborto } \\
\hline 0 & 32 & 57,1 & 16 & 28,5 & \multirow{3}{*}{$0,276^{*}$} \\
\hline 1 & 2 & 3,5 & 4 & 7,1 & \\
\hline 2 & 2 & 3,5 & - & - & \\
\hline \multicolumn{6}{|c|}{ Duração gestação atual (semanas) } \\
\hline$\geq 40 \mathrm{e}<41$ & 16 & 28,5 & 9 & 16 & \multirow{3}{*}{$0,999^{*}$} \\
\hline$\geq 41$ e $<42$ & 16 & 28,5 & 9 & 16 & \\
\hline$\geq 42$ & 4 & 7,1 & 2 & 3,5 & \\
\hline \multicolumn{6}{|c|}{ Consistência do colo } \\
\hline Mole & 18 & 32,1 & 9 & 16 & \multirow{4}{*}{$0,379^{*}$} \\
\hline Grosso & 14 & 25 & 7 & 12,5 & \\
\hline Parcialmente amolecido & 4 & 7,1 & 2 & 3,5 & \\
\hline Sem registro & - & - & 2 & 3,5 & \\
\hline \multicolumn{6}{|l|}{ Posição do colo } \\
\hline Anterior & 1 & 1,8 & 1 & 1,8 & \multirow{4}{*}{$0,028^{*}$} \\
\hline Centralizado & 12 & 21,4 & 12 & 21,4 & \\
\hline Posterior & 23 & 41,2 & 6 & 10,7 & \\
\hline Sem registro & - & - & 1 & 1,8 & \\
\hline
\end{tabular}

Legenda: *Teste exato de Fisher.

Fonte: Oliveira FJS, et al., 2017.

Referente aos dados de antecedentes obstétricos que mostram correlação significativa com o tipo de parto evidenciou-se que as mulheres eram primigestas, nulíparas e evoluíram para parto cesariano. Coura Filho $O$ (2014) constatou a alta incidência de primigestas $(40 \%$ versus $21 \%$ secundidestas) e obesas ( $31 \%$ com $\mathrm{IMC}>30 \mathrm{mg} / \mathrm{m}^{2}$ ) em grupo de 38 gestantes com diagnóstico confirmado de pós-datismo; observações consistentes com estudos anteriores mostraram que pacientes primigestas parecem ter maior predisposição ao pós-datismo quando comparadas com gestantes multigestas.

Outro estudo demonstrou resultados similares, verificando que um grupo de gestantes obesas apresenta uma taxa aumentada de indução do parto que culmina para cesariana ( $28 \%$ versus $19 \%$ para mulheres com peso normal), constatando que uma mulher obesa com gravidez prolongada teria $60 \%$ de chance de parto normal se primípara e 90\% de chance se for multípara (ARROWSMITH S, WRAY S e QUENBYS S, 2011).

As durações das gestações se encontravam entre 40 e 42 semanas. Resultados semelhantes ao estudo descritivo transversal de Girotto (2013) de abordagem quantitativa, que teve como foco caracterizar as gestantes submetidas ao uso de misoprostol para a indução do trabalho de parto, atendidas no Hospital das Clínicas da Faculdade de Medicina de Marília, Unidade II, de Marília-SP, mostram que 44,8\% dos casos de indução do parto com o uso de misoprostol foram atribuídos. Esse código refere-se à gravidez prolongada ou pós-datismo, ou seja, toda gestação com duração maior ou igual a 294 dias ou 42 semanas. 
De acordo com a literatura, várias são as opções utilizadas para se obter atividade uterina de trabalho de parto na tentativa de promover o parto via vaginal. Entretanto, na prática obstétrica do país, as opções se restringem à utilização farmacológica das prostaglandinas e da ocitocina. O estado do colo no início da indução é um dos determinantes mais importantes do curso de eventos subsequentes e é necessário conhecê-lo para estabelecer o procedimento que melhor cumpra esse objetivo (GOMES K, SOUSA AMM e MAMED FV, 2008).

Esse estudo revelou ainda a prevalência de mulheres com colo uterino de consistência amolecida com desfecho para parto cesariano e apresentou significância nas variáveis em relação à posição do colo, em que as mulheres que possuíam colo posterior evoluíram para parto cesariano. Este desfecho é fundamentado pelo conceito de Sampaio ZS et al. (2004), que afirma que as condições cervicais representam um importante fator para o adequado desempenho do método de indução. Em muitas condições clínicas representativas de indicação para indução do trabalho de parto, a cérvice desfavorável é frequentemente encontrada, sobretudo em idade gestacional precoce, o que pode causar falha da indução e consequente aumento da incidência de cesarianas.

Assim, torna-se notório que houve um aumento do número de induções nos últimos anos. No entanto, observa-se que essas induções podem evoluir de forma positiva para o parto normal ou não, considerandose ainda que essa prática necessite de tempo para ser realizada e o desfecho esperado pode se deflagrar em 24 horas ou mais, sendo este tempo uma variável que deve ser valorizada, pois durante esse período a gestante pode demonstrar medo e ansiedade, sentimentos estes que não são benéficos para ela e o bebê; assim, esse intervalo de tempo pode se tornar um ponto negativo. Entretanto o objetivo principal é buscar a evolução para um parto normal, mesmo o contrário ser um desfecho que deve ser considerado. Antes de iniciar o processo de indução ao trabalho de parto, é necessária uma avaliação do bem estar fetal, assim, também se faz essencial a verificação do colo uterino, se o mesmo é favorável ou não para um desfecho de parto normal; essa avaliação é possível pela utilização do índice de Bishop, que utiliza parâmetros que avaliam as condições uterinas. Na Tabela $\mathbf{3}$ a seguir são descritos os registros de prontuários sobre esta variável.

Tabela 3 - Descrição da utilização e registro do índice de Bishop como critério de indução do trabalho de parto. Teresina-PI, $2017(\mathrm{~N}=56)$.

\begin{tabular}{cccccc}
\hline Escore Bishop & $\begin{array}{c}\text { Parto Cesário } \\
\text { № }\end{array}$ & $\%$ & $\begin{array}{c}\text { Parto Normal } \\
\text { № }\end{array}$ & $\%$ & Valor $\mathbf{p}$ \\
\hline Antes da indução & & & & & \\
$<7$ & 3 & 5,3 & 4 & 7,1 & \multirow{2}{*}{$0,133^{*}$} \\
$>7$ & - & - & 1 & 1,8 & \\
Sem registro adequado & 33 & 58,9 & 15 & 26,8 & \\
\hline
\end{tabular}

Legenda: ${ }^{\star} T e s t e$ exato de Fisher. Fonte: Oliveira FJS, et al., 2017.

Nos prontuários nos quais se obteve o registro do índice de Bishop $<7$, evidenciou-se o parto normal, com $7,1 \%$, e a maior parte da amostra, com $58,9 \%$, estava sem registro adequado, e todas evoluíram para parto cesariano. A pesquisa evidenciou que, dos dados que foram registrados adequadamente no prontuário, as pacientes evoluíram para parto normal, no entanto, vale ressaltar que, na maioria dos casos, não havia registro adequado.

Conforme Silva RM (2011), a utilização do Índice de Bishop é de fundamental importância para a definição de qual fármaco utilizar para a indução do parto. Escores no Índice de Bishop abaixo de 6 pedem o uso do misoprostol, pois este fármaco, além de ter ação ocitócita, auxilia nas contrações uterinas e atua na maturação da cérvice, visto que as prostaglandinas agem diretamente nas fibras colágenas do colo do útero separando, dissolvendo e amolecendo as fibras, promovendo, dessa forma, o apagamento e dilatação da cérvice. A avaliação do colo uterino, por meio do índice de Bishop, é relatada pela literatura como uma ferramenta útil no sucesso da indução, se for seguida de forma correta e consistente, embora não se tenha registros adequados a respeito desse processo; no entanto, nas induções analisadas, este fato não mostrou relação significativa com o desfecho final. 
As induções podem ocorrer de forma farmacológica ou não farmacológica, encontrando-se na literatura diversas drogas com suas dosagens e vias de administração; no estudo em questão, foram utilizadas algumas dessas terapias (Tabela 4).

Tabela 4 - Descrição dos fármacos utilizados, doses, via de administração, intervalo entre as doses e tempo de indução, segundo desfecho final. Teresina-PI, 2017 ( $N=56)$.

\begin{tabular}{|c|c|c|c|c|c|}
\hline \multirow{2}{*}{ Variáveis } & \multicolumn{2}{|c|}{ Parto Cesário } & \multicolumn{2}{|c|}{ Parto Normal } & \multirow{2}{*}{ Valor $p$} \\
\hline & № & $\%$ & № & $\%$ & \\
\hline \multicolumn{6}{|l|}{ Indução farmacológica } \\
\hline Misoprostol & 22 & 39,3 & 8 & 14,3 & \multirow{3}{*}{0,167} \\
\hline Ocitocina & 11 & 19,6 & 7 & 12,5 & \\
\hline Misoprostol e ocitocina & 3 & 5,3 & 5 & 8,9 & \\
\hline \multicolumn{6}{|l|}{ Dose em miligramas(mg) } \\
\hline 1 a 4 DOSE 25 & 23 & 41,2 & 9 & 16 & \multirow{4}{*}{$0,508^{*}$} \\
\hline microgramas $(\mathrm{mcg})$ & & & & & \\
\hline 5UI OCITOCINA & 11 & 19,6 & 8 & 14,3 & \\
\hline MISO+OCITOCINA & 2 & 3,5 & 3 & 5,3 & \\
\hline \multicolumn{6}{|l|}{ Via de administração } \\
\hline Vaginal & 25 & 44,6 & 12 & 21,4 & \multirow{2}{*}{0,474} \\
\hline Parenteral & 11 & 19,6 & 8 & 14,3 & \\
\hline \multicolumn{6}{|c|}{ Intervalo entre as doses } \\
\hline 6 horas & 23 & 41,2 & 9 & 16 & \multirow{3}{*}{$0,385^{*}$} \\
\hline 8 horas & 2 & 3,5 & 2 & 3,5 & \\
\hline Contínuo & 11 & 19,6 & 9 & 16 & \\
\hline \multicolumn{6}{|l|}{ Tempo de indução(horas) } \\
\hline$\leq 1$ & 5 & 8,9 & 3 & 5,3 & \multirow{5}{*}{$0,755^{*}$} \\
\hline $2-5$ & 4 & 7,1 & 5 & 8,9 & \\
\hline $6-12$ & 15 & 26,8 & 7 & 12,5 & \\
\hline $13-24$ & 9 & 16 & 4 & 7,1 & \\
\hline$\geq 25$ & 3 & 5,3 & 1 & 1,8 & \\
\hline
\end{tabular}

Legenda: ${ }^{\star}$ Teste exato de Fisher. Fonte: Oliveira FJS, et al., 2017.

A indução farmacológica mais utilizada foi o misoprostol isoladamente, com $57,2 \%$, que foi administrado por via vaginal em $44,6 \%$ das mulheres, com intervalo entre as doses de 6 horas em $41,2 \%$, com tempo de duração de $6-12$ em $26,8 \%$ das mulheres; todas estas evoluíram para parto cesariano, dado que não demonstraram significância em relação às variáveis com o tipo de parto.

As induções utilizadas nas participantes dessa pesquisa foram farmacológicas, utilizando-se o misoprostol isoladamente, e tiveram evolução para parto cesariana. Segundo Feitosa FEL et al. (2006), o fármaco é o método mais efetivo que os convencionais para amadurecer o colo e induzir o parto, associando-se a maior frequência de partos vaginais dentro de 24 horas e menor necessidade de utilização de ocitocina. Relata ainda que estes resultados não são surpreendentes quando se considera que esse medicamento representa, na atualidade, uma alternativa de grande eficácia para induzir o trabalho de parto em gestantes com colo desfavorável.

Foi possível observar que, em relação à evolução do trabalho de parto, a duração em média do início do procedimento até a indicação do parto cesárea foi equivalente a 6-12 horas; dessa forma, as gestantes fizeram o uso de 2 doses de $25 \mathrm{mcg}$, por via vaginal. Diante desta informação, se torna questionável o porquê de a via sublingual não ser utilizada. De acordo com Souza ASR (2010), o uso por via sublingual oferece um maior conforto a paciente, na justificativa de diminuir a quantidade de exames vaginais, além de apresentar uma grande biodisponibilidade em relação à outras vias evitando o mecanismo hepático de primeira passagem devido a sua absorção ocorrer pela mucosa sublingual.

Em relação à indicação do parto cesariano foi evidenciado que prevaleceu o registro de falha de indução, seguido de parada de progressão e sofrimento fetal. O estudo de Rodríguez OF, Martínez IH e Morejón MP 
(2010) consistiu-se de gestantes que deram a luz em uma maternidade de atendimento exclusivo a pacientes oriundas do Sistema Único de Saúde, situada no interior de São Paulo no período de janeiro a junho de 200, uma amostra composta de 119 gestantes submetidas à indução do trabalho de parto com ocitocina, misoprostol, ou ambos, verificou-se que o sofrimento fetal agudo também teve participação importante na indicação do parto cesárea, com $35,1 \%$, acompanhado por desproporção céfalo-pélvica $(23,4 \%)$, falha de indução $(16,2 \%)$ e más condições obstétricas (12,3\%). Indicações ao parto cesário semelhantes ao estudo em questão.

O objetivo de induzir o trabalho de parto é que o mesmo evolua para parto normal da forma mais fisiológica possível, entretanto, nesta pesquisa, o percentual das induções que evoluíram para parto cesariano foi maior que o desfecho de parto normal. A tabela 5 a seguir descreve todas as justificativas de encaminhamento para o centro cirúrgico encontradas nos prontuários das mulheres pesquisadas.

Tabela 5 - Descrição da justificativa de encaminhamento ao centro cirúrgico para desfecho de parto Cesário. Teresina-PI, 2017 ( $\mathrm{N}=56)$.

\begin{tabular}{lcc}
\hline Justificativa de encaminhamento ao centro cirúrgico & No & $\%$ \\
\hline Sofrimento fetal & 5 & 14,8 \\
Falha de indução & 14 & 41,2 \\
Taquissistolia & 1 & 2,9 \\
Parada de progressão & 7 & 20,6 \\
Oligodrâmio severo & 1 & 2,9 \\
Parada da descida secundária & 1 & 2,9 \\
Ctg não tranquilizadora & 1 & 2,9 \\
Periodo expulsivo prolongado & 1 & 2,9 \\
Sem registro específico & 1 & 2,9 \\
Epigastralgia intensa e turvação visual & 2 & 6,0 \\
\hline
\end{tabular}

Legenda: *Teste exato de Fisher. Fonte: Oliveira FJS, et al., 2017.

Da amostra representada por 56 mulheres em processo de indução do trabalho de parto, 60,7\% das gestantes não evoluíram para parto normal, com justificativa prevalente do encaminhamento ao centro cirúrgico com diagnóstico de falha de indução, com $41,2 \%$, seguido por parada de progressão, com $20,6 \%$ e sofrimento fetal, com $14,8 \%$.

O sofrimento fetal encontra-se frequentemente associado ao número excessivo de contrações uterinas, provocando a síndrome de hiperestimulação uterina. Já o desfecho de falha de indução é definida quando o trabalho de parto não é deflagrado, podendo ocorrer em aproximadamente $10 \%$ das gestante a depender do método indução utilizado, ainda em seu estudo Souza ASR et al. (2010) destacam que o recente aumento da prevalência da indução do parto e a falta de consenso da definição da falha de indução pode contribuir para as altas taxas de partos cesário desnecessários.

De acordo com a hipótese desta pesquisa o aumento nas induções do trabalho de parto realizado em gestantes a partir de 40 semanas promove redução do número de cesarianas. Após coleta dos dados e análise das variáveis conclui-se que não se constatou o desfecho das pacientes para parto normal, considerando que no processo de indução existe fatores intrínsecos da gestante e uma questão de sofrimento, demora e ansiedade que está relacionado a duração desse processo.

Conforme a Organização Mundial de Saúde (OMS, 1996), a taxa máxima aceitável de cesáreas em qualquer região é de $15 \%$ e, apesar do uso iatrogênico de cesáreas ser mais frequentemente atribuído a instituições privadas e à população de maior renda (BARBOSA GP, et al., 2003), no presente estudo, conforme já citado anteriormente, foi observada a incidência de $60,7 \%$ de cesarianas, número este muito acima do recomendado pela Organização Mundial de Saúde (OMS).

Importante ressaltar as limitações do presente estudo que consiste na ausência um grupo controle, o tamanho amostral foi pequeno, não houve randomização e o estudo foi de caráter descritivo, observando-se 
grande carência de marcos teóricos que abordassem esta mesma linha de pesquisa, assim como o registro inadequado nos prontuários sobre o processo. Assim, sugere-se que novos estudos sejam realizados com um tamanho amostral maior e a presença de um grupo controle, comparando diferentes métodos de indução e em grupos específicos de pacientes.

\section{CONCLUSÃO}

Conforme os dados, a pesquisa mostra mulheres na idade adulta jovem, solteiras, baixa escolaridade e renda, de ocupação doméstica. O estado civil e a faixa etária evidência que na fase adulta jovem, as taxas de gravidez sem envolvimento conjugal se tornam cada vez mais frequentes e as primigestas não evoluem satisfatoriamente para parto normal, observando-se que estas obtinham o colo favorável, porém de posição posterior, demonstraram significância estatística. Os resultados evidenciaram o parto cesário nas induções com misoprostol devido à frequência de falha de indução, podendo considerar que a posição do colo e primiparidade foram estatisticamente significantes, devido a isso, observa-se a necessidade da existência de um protocolo a ser seguido por toda equipe envolvida no processo de indução. O resultado mostrou que a maioria apresentou falhas, fato que pode estar relacionado ao desfavorecimento do colo na posição posterior, sendo que é possível na prática clínica, avaliar o colo na própria admissão, para saber a real probabilidade de se atingir um desfecho favorável, podendo evitar procedimentos desnecessários. A relevância deste estudo se observa a partir da análise do aumento no número de induções ao trabalho de parto no país, onde esse processo ocorre na tentativa de reduzir as taxas de parto cesário.

\section{REFERÊNCIAS}

1. ACOG. Practice bulletin no. 43: Management of preterm labor. Obstetric sand Gynecology, 2012; 119(6): $1308-1317$.

2. APPOLINÁRIO F. Metodologia da ciência: filosofia e prática da pesquisa. São Paulo. Cengage Learning, $2012 ; 2$ : 226.

3. ARROWSMITH S, et al. Maternal obesity and labour complications following induction of labour in prolonged pregnancy. BJOG: an international journal of obstetrics and gynaecology, 2011; 118(5): 578-588.

4. AUSTIN S, et al. Labor induction with intravaginal misoprostol compared with the dinoprostone vaginal insert: $A$ systematic review and metaanalysis. American Journal of Obstetrics \& Gynecology, 2010; 116: 450-463.

5. BARBOSA GP. et al. Parto cesáreo: quem o deseja? Em quais circunstâncias? Cad., Saúde Pública, Rio de Janeiro, 2003; 19(6): 1611-1620.

6. BRASIL. Ministério da Saúde. Secretaria de Atenção à Saúde. Departamento de Ações Programáticas Estratégicas. Gestação de Alto Risco: manual técnico/ Ministério da Saúde, Secretaria de Atenção à Saúde, Departamento de Ações Programáticas Estratégicas. Brasília: Editora do Ministério da Saúde, 2010; 5:302.

7. CARVALHO SA. A importância do parto Normal para mulheres. Trabalho de Conclusão de Curso apresentado ao Curso de Especialização em Atenção Básica em Saúde da Família. Universidade Federal de Minas Gerais, Campos Gerais-MG, 38 fls. 2013.

8. CARVALHO VF, et al. Práticas prejudiciais ao parto: relato dos trabalhadores de saúde do sul do Brasil. Revista da Rede de Enfermagem do Nordeste - Revista Rene, 2010; 11: 92-98.

9. CAMARGO LA, et al. Antropometria e aspectos neonatais de recém-nascidos de gestações de 41 a 42 semanas. Revista da Faculdade de ciências médicas. Sorocaba, 2014; 16(4): 182-187.

10. CHAVES NETTO H, SÁ RAM. Obstetrícia Básica. 3 ed. São Paulo: Atheneu, 2015.

11. COURA FILHO O. Gravidez prolongada. In: Rezende, JED. Obstetícia.13 ed. Rio de Janeiro, Guanabara Koogan, 2014.

12. CORREGIO KS, et al. Comparação entre dinoprostone e misoprostol na indução do trabalho de parto em gestações pós-data na presença de cérvix desfavorável. Revista Arquivo Catarinense de Medicina, 2014; 4(2): 23-28.

13. CHAVES NETTO H, et al. Manual de condutas em Obstetrícias. 3 ed. São Paulo: Atheneu, 2011.

14. CHENG YW, et al. Indução do trabalho de parto em relação ao manejo expectante em mulheres de baixo risco e resultados perinatais associados. Am J ObstetGynecol, 2012; 207(6):502

15. CHESTNUT D, et al. Anestesia obstétrica: princípios e prática. 5 ed. Rio de Janeiro: Elsevier, 2016.

16. CLARK SL, et al. The Clinical and Economic Impact of Nurse to Patient Staffing Ratios in Women Receiv in Intra partum Oxytocin. American Journal of Perinatology. New York, 2014; 31(2). 
17. DOMINGUES RQ. O uso da ocitocina exógena no trabalho de parto: uma revisão integrativa. Monografia apresentada ao curso de especialização em Enfermagem Obstétrica- Rede Cegonha- Universidade Federal de Minas Gerais, Escola de Enfermagem, para obtenção do título de especialista em Enfermagem Obstétrica. Porto Alegre/RS. 2016.

18. EHRENTHAL D, et al. Labor induction and the risk of a cesarean delivery among nulliparous women at term Obstetrics and Gynecology. Obstetrics and Gynecology, 2010; 116(1): 35-42.

19. FEITOSA FEL, et al. Nova formulação de misoprostol sublingual na indução do trabalho de parto. Rev. Assoc. Med. Bras, São Paulo, 2006; 52(4): 251-255.

20. FREITAS F, et al. Rotinas em Obstetrícia. 6. ed. Porto Alegre: Artmed, 2011.

21. GATTÁS DSMB, et al. Baixa dose de misoprostol sublingual $(12,5 \mu \mathrm{g})$ para indução do parto. Rev. Bras. Ginecol. Obstet., Rio de Janeiro, 2012; 34(4): 164-169.

22. GIROTTO LC, et al. Gravidez e misoprostol: caracterização das gestantes atendidas em um hospital escola do interior de São Paulo. Rev. Bras. Pesq. Saúde. Vitória, 2013; 15(3): 45-52.

23. GOLDEBERG AE. Cervical ripeninge. Medscape. 2015.

24. GOMES K, SOUSA AMM, MAMEDE FV. Indução do trabalho de parto em primíparas com gestação de baixo risco. Rev. Eletr. Enf., 2010; 12(2): 348-353.

25. IBGE. Instituto Brasileiro de Geografia e Estatística, Censo 2010.

26. KING TL, BRUCKER MC. Pharmacology for women's Healt. Sudbury, MA: Jones and Bartlett Publishers, 2011.

27. KNUPP VMA, et al. Distribuição do parto vaginal e da cesariana no município do rio de janeiro no período de 2001 a 2004. Rev Enferm.,2008; 12(1): 39-44.

28. LOWDERMILK DL, et al. Saúde da Mulher e Enfermagem Obstétrica. 10.ed. Rio de Janeiro: Elsevier, 2012.

29. MANDARINO NR, et al. Aspectos relacionados à escolha do tipo de parto: um estudo comparativo entre uma maternidade pública e outra privada, em São Luís, Maranhão, Brasil. Cad. Saúde Pública, 2009; 25(7): $1587-1596$.

30. MARCONI MA, LAKATOS EM. Metodologia do Trabalho Científico. 8. ed. São Paulo. Editora: Atlas, 2017.

31. MONTENEGRO CAB, REZENDE FILHO J. Obstetrícia fundamental. 13. ed. Rio de Janeiro: Guanabara Koogan, 2015.

32. MOREIRA BR, et al. Determinantes relacionados ao parto cesáreo em maternidade pública da Região Metropolitana de Belo Horizonte - MG. Rev. Med. Minas Gerais, Minas Gerais, 2016; 26.

33. NASCIMENTO MI, et al. Misoprostol use under routine conditions for termination of pregnancies with intrauterine fetal death. Rev. Assoc. Med. Bras., São Paulo, 2013; 59(4).

34. PEIXOTO KD. A Implementação das Práticas de Indução do Trabalho de Parto em um Hospital Universitário. Porto Alegre, 2013.

35. PUNDEK MRZ, ROHRBACHER I. Métodos terapêuticos de maturação cervical. Femina, Santa Catarina, 2013; 41(2): 90-92.

36. QUEIROZ MVO, et al. Incidência e características de cesáreas e de partos normais: estudo em uma cidade no interior do Ceará. Rev. Bras. Enferm., 2005; 58(6): 687-691.

37. RICCI SS. Enfermagem Materno Neonatal e Saúde da Mulher. 3. ed. Rio de Janeiro: Guanabara Koogan, 2015.

38. RODRIGUEZ OF, et al. Inducción del parto con oxitocina, prostaglandinas o ambas. Rev. Cubana Obstet. Ginecol., $2001 ; 27(2): 135-140$.

39. SANTOS I, RAMALHO C. Indução eletiva do trabalho de parto às 39 semanas de gestação vs atitude expectante: revisão sistemática. Acta Obstet Ginecol Port., 2016; 10(3).

40. SILVA RM, et al. A efetividade do misoprostol e da ocitocina nos diferentes períodos do parto. Rev. Enferm., LondrinaPR, 2011; 5(6): 1549-554.

41. SAMPAIO ZS, et al. Fatores associados ao parto vaginal em gestantes de alto risco submetidas à indução do parto com misoprostol. Rev. Bras. Ginecol. Obstet., 2004; 26(1): 21-29.

42. SIMPSON KR. Clinicians' guide to the use of oxytocin for labor in Bduction and argumentation. Journal of Midwifery e Women's Healtb., 2011; 56(3): 214-221.

43. SESAPI - Secretaria de Estado da Saúde do Piauí.

44. SOUSA ASR, et al. Métodos farmacológicos de indução do trabalho de parto: qual o melhor? Femina,2010; 28(5).

45. SOUZA GN, et al. Métodos de indução do trabalho de parto. Femina, 2013; 41(1): 48-54.

46. SOUZA ASR. Análise crítica dos métodos não-farmacológicos de indução do trabalho de parto. Femina, 2010; 38(4): 196-201.

47. WOJNAR DM, et al. Outcomes of the Evidence-Based Pitocin Administration Checklist at a Tertiary-Level Hospital. Western Journal of Nursing, 2014; 36(8):975-988. 